\title{
Currículo Baseado em Competências na Residência Médica
}

\author{
Competency-Based Curriculum in Medical \\ Residency
}

\author{
Cláudia Regina Fernandes \\ Alberto Farias Filho ${ }^{I I}$ \\ Josenilia Maria Alves Gomes ${ }^{I}$ \\ Washington Auspilicueta Pinto Filho ${ }^{I}$ \\ Glauco Kleming Florêncio da Cunha ${ }^{\text {III }}$ \\ Flavio Lobo Maia ${ }^{I V}$
}

\section{PALAVRAS-CHAVE:}

- Currículo.

- Educação Baseada em Competências.

- Internato e Residência Médica.

- Competência Profissional.

- Avaliação Educacional.

KEYWORDS:

- Curriculum.

- Competency-Based Education.

- Internship and Residency.

- Professional Competence.

- Educational Measurement.

Recebido em: 25/01/2011

Reencaminhado em: 15/06/2011

Aprovado em: 04/10/2011

REVISTA BRASLIEIRA DE EDUCAÇ̄̃o MÉdica $129 \frac{11}{36(1): 129 \cdot 136 ; 2012}$
${ }^{I}$ Universidade de Fortaleza, Fortaleza, CE, Brasil.

${ }^{I I}$ Universidade Federal do Ceará, Fortaleza, CE, Brasil.

III Prefeitura Municipal de Fortaleza, Instituto Dr José Frota, Instituto Dr José Frota, Fortaleza, CE, Brasil.

IV Universidade Estadual do Ceará, Fortaleza, CE, Brasil. 


\section{INTRODUÇÃO}

No Brasil, os cursos de graduação em Medicina devem seguir as diretrizes curriculares nacionais propostas pelo Ministério da Educação (DCN, 2000) ${ }^{1}$. Entretanto, para os diversos programas de residência médica, não existem diretrizes curriculares nacionais propostas, tampouco exigências de processos de avaliação para certificação ao final de treinamento do especialista. Na América do Norte, um movimento liderado pelo The Royal College of Physicians and Surgeons of Canada (RCPSC) ${ }^{2}$ e pelo American Accreditation Council on Graduate Medical Education $(\mathrm{ACGME})^{3}$ propõe alterações no currículo médico de pós-graduação, para garantir que os programas de treinamento de especialistas sejam responsivos às necessidades dos sistemas público e privado de saúde e da sociedade em geral. Na mesma direção apontam universidades europeias, como Dundee, na Escócia, e Maastricht, na Holanda. A ênfase destes novos programas propostos está no profissionalismo como a regra, trabalhando numa definição ampla, que vai além do técnico, baseando-se em competências, o que remete à necessidade inicial da definição de competências.

De acordo com Albanese e colaboradores, a falta de características precisas que definam claramente o que realmente constitui uma competência suscita dúvidas e discussões ${ }^{4}$. Competência foi inicialmente definida como uma síntese de conhecimentos, habilidades e atitudes que, integrados, propiciam ao indivíduo aprimorar a utilização dos recursos cognitivos e técnicos para diagnosticar, tratar e proporcionar benefício, menor morbidade ao doente e menor custo às instituições. Tais recursos devem ser coerentes com a ética profissional, com a melhor evidência científica disponível e com a experiência pessoal. Govaerts amplia um pouco mais o espectro da definição quando afirma que competências dependem do contexto, além de demandarem experiência, reflexão sobre a própria prática e capacidade de continuar aprendendo por toda a vida ${ }^{5}$. A despeito da ausência de uma única definição suficientemente abrangente, o desenvolvimento de competências é fundamental para o desempenho do profissional frente às constantes mudanças que ocorrem durante sua prática.

Os indicadores de competência são parâmetros que possibilitam a observação e comprovação do exercício desta e implicam a avaliação integrada das três dimensões: conhecimentos, habilidades e atitudes. Ou seja, avaliar a aquisição de competências implica, portanto, a utilização de múltiplos instrumentos de avaliação que sejam capazes de proporcionar, juntos, uma visão real da aquisição.

A construção de um currículo baseado em competências implica necessariamente a construção de um sistema de avaliação confiável e válido, contendo elementos variados, incluindo testes escritos, observação em cenários de prática e do comportamento profissional verificado pelas atitudes. Salienta-se que a avaliação, neste contexto, obriga o feedback construtivo do avaliador.

Baseados nos conceitos expostos e na necessidade de aprimorar o perfil do anestesiologista egresso, os preceptores do programa de residência médica em Anestesiologia do Hospital Universitário Walter Cantídio (HUWC) da Universidade Federal do Ceará, em janeiro de 2007, assessorados por um consultor, formaram um grupo de trabalho com o objetivo de reestruturar aquele programa, definindo as competências a serem desenvolvidas. O produto do trabalho deste grupo deu corpo a um novo formato de programa, embasado em um currículo delineado nos três anos, com instrumentos de avaliação multidimensionais.

Tomando como referenciais teóricos estudos sobre competências em Anestesiologia ${ }^{2-6}$, contextualizados pelo cenário próprio do serviço de um hospital universitário público e terciário, foram identificadas seis competências principais. $\mathrm{O}$ passo subsequente foi listar os conhecimentos, habilidades e atitudes requeridos para a aquisição de cada uma das competências.

O objetivo deste artigo é descrever como foi desenvolvido o currículo implantado na residência de Anestesiologia do Hospital Universitário Walter Cantídio da Universidade Federal do Ceará, além de demonstrar os instrumentos utilizados no sistema de avaliação.

\section{METODOLOGIA}

\section{Descrição do Processo de Trabalho}

O ponto inicial foi definir as competências, a partir de um perfil de excelência em Anestesiologia que deveria ser demonstrado pelos egressos do programa. Foi formado um grupo de trabalho composto pelos preceptores do serviço. O primeiro momento caracterizou-se pela discussão de evidências, vantagens e desvantagens do embasamento de um currículo por competências. Todos os preceptores participantes fizeram ou fazem gestão de serviços de Anestesiologia em hospitais públicos, e a pergunta norteadora utilizada para iniciar o processo de discussão foi: qual o perfil do anestesiologista que o serviço público necessita? As respostas foram variadas e incluíram: um anestesista compromissado com o serviço; capaz de se relacionar bem com a equipe; capaz de inovar; portador de atitude positiva e integradora; capaz de elaborar e cumprir protocolos; capaz de utilizar racionalmente fármacos e tecnologias; que tenha a mente aberta e aceite mudanças; capaz de elaborar fichas de anestesia completas; e que seja tecnicamente eficiente.

Com o intuito de facilitar a compilação das características relacionadas a partir da discussão do grupo de trabalho, optou-se pela sistematização, agrupando as competências em 
três grandes eixos: o eixo humanístico e documental (competências 1 e 2), o técnico-científico (competências 3, 4 e 5) e o de qualidade em serviço (competência 6).

Na concepção dos autores, o eixo humanístico documental e o da qualidade em serviço permeiam continuamente o técnico-científico e, portanto, são partes integrantes de todos os cenários de prática e processos de avaliação. A interação entre estes eixos visa formar um anestesiologista que, além de profundo conhecedor dos procedimentos técnicos e fármacos, tenha uma atitude crítica e reflexiva e seja capaz de se manter estudando e se atualizando durante sua vida profissional e, desta forma, possa interagir com o serviço onde está inserido, modificando-o e funcionando como agente de melhorias, quando necessário.

Para cada uma das competências listadas foram descritos os conhecimentos necessários para construí-la e as habilidades que, de forma prática, podem ser demonstradas e avaliadas.

\section{DEFINIÇÃO DAS COMPETÊNCIAS}

\section{Competência 1 - Comunicação}

Duas dimensões devem ser observadas na avaliação desta competência:

Relação anestesiologista-paciente: quando desenvolvida, esta competência permite o acolhimento do paciente cirúrgico tendo como princípios básicos a responsabilização e criação de vínculo. Demanda do anestesiologista uma visão sistêmica do paciente, indo além da doença cirúrgica deste e das comorbidades. Visa estabelecer uma relação de confiança por meio do conhecimento e do emprego de técnicas de linguagem verbal e não verbal.

Relação interprofissional: desenvolvimento de relações interprofissionais embasadas no respeito às peculiaridades dos indivíduos e das especialidades envolvidas no cenário perioperatório e à hierarquia.

Os conhecimentos e habilidades elencados para desenvolvimento desta competência são:

CONHECIMENTOS Técnicas de comunicação verbal e não verbal; noções de psicologia médica; Código de Ética Médica; organograma dos níveis hierárquicos do serviço; direitos e deveres dos profissionais que atuam conjuntamente.

HABILIDADES - Comunicar-se de forma clara e assertiva;

- Reduzir, pelo acolhimento, a ansiedade pré-operatória, reconhecendo o impacto emocional que uma doença cirúrgica e o procedimento anestésico-cirúrgico causam no paciente;

- Reportar-se adequadamente à equipe e à família em situações de desfechos indesejáveis;

- Demonstrar espírito de liderança.

\section{Competência 2 - Documentação}

Registro adequado dos procedimentos, intercorrências e evolução do paciente no período perioperatório.

CONHECIMENTOS Legislação específica; normas e rotinas da instituição; código de ética médica; padrão de preenchimento dos registros.

HABILIDADES Registro dos procedimentos técnicos de forma legível e sistemática, devidamente datados durante o período perioperatório.

\section{Competência 3 - Cuidados Pré-Operatórios}

Avaliação pré-operatória, com estratificação e proposta de manejo adequado do risco anestésico-cirúrgico.

CONHECIMENTOS Consulta pré-operatória e pré-anestésica; estratificação de risco anestésico-cirúrgico; manejo pré-operatório de morbidades cardiovasculares e respiratórias mais prevalentes, farmacologia das medicações pré-anestésicas.

HABILIDADES • Realizar avaliação clínica do paciente cirúrgico no pré-operatório;

- Prescrever corretamente medicação préanestésica;

- Solicitar, de forma racional, exames préoperatórios, baseando-se nas melhores evidências, obedecendo a princípios éticos, bioéticos e legais;

- Reconhecer a necessidade de interconsulta com outro especialista;

- Planejar a técnica anestésica integrando a condição clínica do paciente e a complexidade da cirurgia.

\section{Competência 4 - Execução do Ato Anestésico - Cuidados Intraoperatórios}

Condução do ato anestésico, resolvendo o problema do paciente com responsabilidade e segurança, sem causar dano adicional.

CONHECIMENTOS Técnica de anestesia geral, bloqueios neuroaxiais e locorregionais; farmacologia dos agentes usados em anestesia; monitorização invasiva e não invasiva; terapia de reposição volêmica e transfusional; uso racional de tecnologias em saúde.

HABILIDADES

- Realizar os procedimentos técnicos inerentes à especialidade; desenvolver o raciocínio clínico e a capacidade de tomar decisão rapidamente;

- Realizar punção venosa central e arterial;

- Interpretar dados de monitorização invasiva; 
HABILIDADES • Realizar reposição volêmica, de hemocomponentes e hemoderivados racionalmente;

- Manejar apropriadamente situações de crise;

- Utilizar corretamente os fármacos anestésicos.

\section{Competência 5 - Cuidados Pós-Operatórios}

Acolhimento do paciente no ambiente de recuperação pós-operatória, mantendo a homeostase, o controle da dor e decidindo o momento mais apropriado para a alta.

Esta competência se desenvolve em duas áreas de atuação: a sala de recuperação pós-anestésica e a unidade de cuidados intensivos pós-operatórios.

CONHECIMENTOS Complicações do período pós-operatório; escala de Aldrete e Kroulik modificada ${ }^{7}$; escala visual analógica da dor; escala de avaliação da sedação e agitação (Richmond) ${ }^{8}$; escala de avaliação do delirium - CAM-ICU (Confusion Assessment Method in a Intensive (are) $)^{8}$; modalidades de ventilação mecânica; critérios clínicos e laboratoriais de extubação; uso de fármacos vasoativos; fármacos para sedação, analgesia e antieméticos.

HABILIDADES Idênticas às descritas para o manuseio do paciente no intraoperatório, adicionadas das seguintes:

- Aplicar as escalas de avaliação da dor e Aldrete Kroulik ${ }^{7}$.

- Identificar precocemente distúrbios cognitivos;

- Tratar dor aguda;

- Manusear o aparelho de ventilação mecânica;

- Prescrever antibióticos e fármacos vasoativos;

- Realizar reanimação cardiopulmonar.

\section{Competência 6 - Gestão de Qualidade e Segurança no Perioperatório}

Controle dos processos para garantia de resultados assistenciais de excelência.

CONHECIMENTOS Legislação específica; noções de custobenefício, fármaco-economia, gerenciamento de risco, padronização de cirurgia segura ${ }^{9}$, indicadores de qualidade e segurança em Anestesiologia ${ }^{10}$.

HABILIDADES Desenvolver metacognição; prática reflexiva, autoavaliação, disciplina e padronização sistemática de rotinas.

\section{Atitudes}

As atitudes não foram individualizadas para cada competência, mas descritas em conjunto como características a serem desenvolvidas em todos os residentes no decorrer do treinamento. São fundamentais e continuamente exigidas no desempenho das atividades diárias. Constituem o cerne do currículo. Desenvolvê-las é o maior desafio do programa, o que realmente diferencia e destaca o profissional, pois se compreende que a Anestesiologia é uma especialidade médica peculiar, sempre por "trás da cena". O anestesiologista cuida de um paciente durante um período de tempo relativamente curto e que é primariamente visto e referenciado por um cirurgião. A anestesia envolve a incorporação de monitores e procedimentos no cuidado ao paciente. Todos estes fatores podem distanciar e mecanizar a relação médico-paciente, propiciando o desenvolvimento de profissionais distantes e pouco comprometidos $^{11}$.

Atitudes a serem observadas e desenvolvidas durante o programa: responsabilização, empatia, assertividade, proatividade, visão integral do paciente, comprometimento, resiliência, respeito às normas e rotinas institucionais, organização, respeito aos níveis hierárquicos, compaixão, confiabilidade, calma em situações de crise, honestidade, disciplina, bom senso, reconhecimento de erros, pontualidade, estabilidade emocional, determinação, estabelecimento de prioridades, boa comunicação, consistência, trabalho em equipe, meticulosidade, criatividade, bom relacionamento, adaptabilidade, curiosidade científica, receptividade, sinceridade, eficiência, educação, autoestima elevada, espontaneidade, senso de humor, discrição, confidencialidade, altruísmo, solidariedade.

\section{Sistema de Avaliação}

A avaliação desempenha um papel central no processo de ensino e aprendizagem. Deve possuir critérios de validade e confiabilidade que assegurem a aquisição das competências estipuladas para o estudante naquele período de estudo ${ }^{12,13}$. Para garantir estas características, o mais adequado é utilizar múltiplos instrumentos. De acordo com Dijkstra et al. ${ }^{14}$, independentemente do valor psicométrico e do ponto de corte utilizado, um único instrumento de avaliação será insuficiente para a aferição de competências como um todo. Partindo-se do pressuposto de que uma competência médica não constitui a soma de entidades isoladas, mas, sim, a integração dos saberes para realizar, é lógico concluir que um instrumento sozinho, apesar do valor psicométrico adequado, não será capaz de fornecer informações sobre a aquisição de uma competência em todos os domínios envolvidos ${ }^{15}$. 
Desta forma, o sistema de avaliação do programa de residência do HUWC foi organizado utilizando-se vários instrumentos: teste cognitivo, instrumento de avaliação de habilidades procedurais por observação direta (Dops), e feedback de múltiplas fontes (MSF).

O teste cognitivo é realizado a cada três meses, com questões objetivas e subjetivas, baseando-se em casos clínicos para avaliar o manejo perioperatório. A correção é realizada em uma reunião aberta entre os residentes e preceptores, o que proporciona feedback imediato através da discussão das respostas esperadas. Feedbacks individuais são também fornecidos.

A avaliação das habilidades técnicas e procedurais é feita mediante observação direta da prática, com o emprego de instrumentos padronizados, que contêm uma lista de conferência para cada procedimento específico. Todos os instrumentos são entregues ao residente no início do ano letivo. As avaliações são efetuadas pelos preceptores previamente treinados para isto, durante a realização do procedimento no ambiente de treinamento. $\mathrm{O}$ formato escolhido é semelhante ao Dops ${ }^{16}$, com feedback construtivo imediatamente ao final da avaliação ${ }^{17}$. O residente é informado previamente sobre o dia de sua avaliação. As listas de conferência (checklists) de cada avaliação são registradas e encadernadas. Assim, ao longo dos três anos, o residente compõe um portfólio de avaliação longitudinal em ambiente de prática clínica, propiciando a observação do padrão de evolução das habilidades técnicas ao longo do treinamento da residência médica.

A avaliação das atitudes constitui, indubitavelmente, o maior desafio. É fato a inexistência de um instrumento fidedigno para avaliação e julgamento adequado de ações. A existência de uma série de regras e normas de conduta no serviço não implica necessariamente a incorporação destas pelos anestesiologistas em formação. Optou-se, para o programa, pela Avaliação de Múltiplas Fontes $\left(360^{\circ}\right)^{18,19}$, aplicada semestralmente. Respondem ao questionário: um cirurgião e uma enfermeira do centro cirúrgico, a secretária do serviço de Anestesiologia, uma técnica de enfermagem da anestesia, a médica intensivista chefe da UTI pós-operatória e uma enfermeira da UTI pós-operatória. Os itens do instrumento para avaliação $360^{\circ}$ sofrem alterações de acordo com o avaliador.

O sistema de avaliação é complementado por um logbook simplificado com conteúdo pré-formato em folha padrão. Nele, o período perioperatório de cada procedimento anestésico realizado pelo residente é anotado. As anotações não se limitam à mera coletânea dos procedimentos realizados. Incluem todos os dados de avalição pré-operatória, planejamento da técnica anestésica e visita pós-operatória e comentários acerca do que foi desenvolvido adequadamente e o que pode ser melhorado.
Ser competente requer a aplicação do conhecimento no fazer das tarefas reais diárias, e os logbooks permitem a documentação da aquisição da competência e o aprendizado reflexivo, que constitui a chave do desenvolvimento profissional ${ }^{20,21}$.

Os casos anotados nos logbooks são discutidos com os preceptores em reunião semanal, permitindo uma reflexão sobre as lacunas na aprendizagem e as necessidades de remediação, além da influência das decisões tomadas durante o ato anestésico no desfecho.

A avaliação do programa e do desempenho dos preceptores é realizada anualmente, mediante instrumento ${ }^{13}$ apropriado, adaptado à realidade do serviço.

\section{Treinamento de Preceptores}

Outro aspecto que merece destaque na descrição do processo de implantação deste currículo é a formação de preceptores. Os responsáveis pelo CanMED ${ }^{1}$, precocemente, no processo de implantação, perceberam as dificuldades dos preceptores em ensinar e avaliar baseando-se em competências. A falta de preceptores preparados para um novo formato constitui um ponto crítico que merece um olhar diferenciado, pois não existe processo de mudança de qualidade do perfil do egresso sem investir naquele que o está formando e que, no ambiente da residência médica, mais do que durante o curso médico, é o seu modelo. A figura do preceptor, portanto, se reveste de uma importância crucial no processo de formação de bons especialistas. A principal função do preceptor é ensinar a clinicar, por meio de instruções formais e com determinados objetivos e metas. Portanto, ele interage fundamentalmente com o aprendiz no cenário da prática clínica diária. Desta forma, suas habilidades técnicas no desempenho de funções clínicas e procedurais são de fundamental importância. Entretanto, suas funções não podem ficar restritas a simplesmente ensinar ou demonstrar a técnica. Eles precisam aconselhar, inspirar e influenciar o desenvolvimento profissional e pessoal dos recém-graduados. Além disso, as avaliações formais também fazem parte da preceptoria ${ }^{22}$.

Para proporcionar o acompanhamento longitudinal dos residentes por uma equipe de preceptores treinados, foi criado um Núcleo de Educação em Anestesiologia (NEA), que reúne os preceptores semanalmente para desenvolvimento em temas docentes atuais de destaque: aprendizagem significativa, feedback construtivo e elaboração de avaliações práticas e testes escritos, além de atualizações na especialidade.

\section{DISCUSSÃO}

O termo currículo se origina da palavra latina currere, de correr. Por definição, portanto, um currículo do programa de re- 
sidência em Anestesiologia deve ser montado com base em ações e experiências pelas quais os recém-graduados devem passar no intuito de transformá-los em anestesiologistas qualificados. Pensar o currículo desta forma garante que o egresso não está totalmente formado, incorporando e levando consigo o conceito de aprender por toda a vida ${ }^{23}$.

Inovação significa fazer as coisas de uma nova forma, proporcionando mudanças em um produto ou organização. Portanto, um currículo inovador deve olhar para o futuro, investindo na formação de indivíduos flexíveis às mudanças, característica imprescindível no cenário médico atual, onde estas acontecem muito rapidamente. É salutar também que este currículo favoreça o desenvolvimento acadêmico dos residentes que almejam seguir carreira na universidade. Os egressos devem ser preparados para buscar constantemente a excelência na prática clínica e/ou acadêmica.

Para muitos, falar de competências para Anestesiologia pode significar uma mera listagem de procedimentos, uma vez que durante muito tempo a especialidade foi descrita como essencialmente técnica, sem preocupação com o paciente e sem atuação além da sala de cirurgia. Somam-se a essa tradição os avanços crescentes da tecnologia e dos fármacos, que direcionam a prática para o extremo tecnicismo. Por outro lado, o crescente desenvolvimento da especialidade desafia o profissional, motivando-o a buscar uma formação mais abrangente. Atualmente são crescentes as oportunidades e a necessidade de atuação em outros espaços além do centro cirúrgico, tais como ambulatórios de avaliação pré-operatória, clínica de dor e cuidados paliativos, centro cirúrgico ambulatorial, unidades de reanimação e pronto atendimento móvel, unidades de emergência, salas de recuperação pós-operatória, terapia intensiva, além da gestão de serviços e carreira acadêmica, pesquisa e ensino com simulação.

Outro conceito a ser superado é o de que o anestesiologista não precisa se comunicar. De acordo com Kopp e Schafer ${ }^{24}$, o anestesiologista se depara com desafios únicos de comunicação em sua prática, que envolvem: pouco tempo de contato para firmar a relação médico-paciente, extrema ansiedade do paciente no momento pré-cirúrgico, associada ao medo da dor, da mutilação, da morte e, finalmente, pacientes com consciência comprometida que impedem a adequada comunicação. Neste contexto, as dificuldades são ainda maiores quando a comunicação está relacionada a desfechos indesejáveis. Outra consideração referente à comunicação, extremamente vinculada à especialidade, é a de trabalhar em equipe. Não existe anestesia sem cirurgia. Portanto, é imprescindível desenvolver competência relacionada à comunicação interprofissional, revestindo-a de preceitos éticos, sobretudo quando o primeiro contato com a equipe se dá imediatamente antes do procedimento ou em uma situação de emergência.

A anestesia é parte integrante de grande número de procedimentos cirúrgicos e diagnósticos. Assim, buscar a qualidade total é fundamental no controle do processo anestésico. Tal conceito é recente e esbarra ainda na falta da descrição de indicadores precisos, a despeito do grande número de publicações sobre o assunto ${ }^{8,16,17}$. Independentemente do indicador utilizado, a segurança é uma das dimensões da qualidade que mais impacta, pois corresponde à maior expectativa dos pacientes em relação ao procedimento anestésico. A gestão da qualidade constitui um instrumento valioso na evolução para a medicina perioperatória, onde o anestesiologista contribui efetivamente para o desfecho do caso ${ }^{25,26}$. Portanto, é essencial estabelecer a gestão da qualidade como integrante das competências a serem desenvolvidas pelo residente de Anestesiologia durante o seu processo de formação. Outro ponto importante a considerar na questão da segurança é a utilização das listas de conferências padronizadas (checklists), que proporcionam, de forma simples e com baixo custo, a redução das complicações e mortes nas salas de cirurgia ${ }^{27}$.

Formar profissionais com base em competências impõe mudanças radicais no processo de avaliação, não apenas no formato, mas sobretudo na concepção. É preciso transformar a cultura da avaliação como instrumento de classificação e punição para investir na avaliação como recurso de aprendizagem significativa, através do feedback construtivo e da revisão dos processos que levaram a um resultado insatisfatório. Os egressos deste programa devem ser capazes de avaliar para o crescimento próprio e do serviço onde estão inseridos. Segundo Haller et al. ${ }^{10}$, qualidade e segurança em anestesia devem ser monitorizadas pela análise da morbimortalidade e dos incidentes no período perioperatório. A revisão do portfólio em reuniões semanais entre os preceptores e os residentes promove o desenvolvimento da reflexão crítica sobre os atos anestésicos realizados, verificando as complicações e identificando os pontos que demandam melhorias. Neste momento, os processos são revistos, e as falhas identificadas, propiciando adequação das rotinas em busca da promoção de segurança e, ao mesmo tempo, disseminando a cultura, entre os anestesiologistas em formação, da reflexão e análise da prática como instrumentos de aprendizagem baseados na própria experiência ${ }^{28}$. O formato padronizado e simplificado do logbook facilita a adesão dos residentes, pois da experiência com logbook na graduação se concluiu que uma grande dificuldade na utilização desta ferramenta é a energia e o tempo dispensados em sua confecção ${ }^{29}$. 
Finalmente, é importante ressaltar a utilização dos princípios educacionais básicos para implementar um programa educacional de formação de especialistas baseado em competências. Ou seja, simplesmente identificar domínios nos quais o anestesiologista deve ser competente não é suficiente. $\mathrm{O}$ real desafio para aqueles envolvidos na concepção de um programa educacional baseado em competências é acompanhar a aquisição do conhecimento, das habilidades e das atitudes que devem ser executadas em cada domínio no nível predeterminado e reconhecer que a performance esperada vai variar de acordo com o estágio em que o residente se encontra e a forma como cada um aprende ${ }^{30}$. Ou seja, formar com base em competências exige acompanhamento contínuo do desenvolvimento do aprendiz, com o intuito de transformá-lo, construí-lo como profissional de excelência, com formação humanística, reflexiva, com senso de responsabilidade social e atuação voltada ao contexto em que vive ${ }^{31}$.

\section{CONCLUSÃO}

A implantação de um programa de residência que vise à formação de especialistas com base na aquisição de competências predefinidas e adequadamente avaliadas é um desafio. Se este programa atingirá os objetivos aqui propostos ainda não está estabelecido. O tempo e a avaliação constante do programa em si, assim como do desempenho profissional dos egressos, irão subsidiar a resposta a este e a outros questionamentos. Os resultados observados na utilização deste formato de currículo na graduação são encorajadores.

É fato que a vigente ausência de padronização nos programas de residência em Anestesiologia torna mais difícil atingir a inovação e o padrão de excelência que irão assegurar o futuro da especialidade dentro e fora do centro cirúrgico.

\section{REFERÊNCIAS}

1. Brasil. Ministério da Educação. Conselho Nacional de Educação. Câmara de Educação Superior. Resolução CNE/CES nº 4, de 7 de novembro de 2001. Institui Diretrizes Curriculares Nacionais do curso de graduação em Medicina Diário Oficial da União. Brasília, 9 nov. 2001; Seção 1, p.38

2. Royal College of Physicians and Surgeons of Canada (CanMEDS). CanMEDS 2000 Project Societal Needs Working Group Report. Med Teacher. 2000;22(6):549-54.

3. Accreditation Council for Graduate Medical Education (ACGME). Outcome Project [online]. 2001. Disponível em www.acgme.org/outcome/comp/compFull.asp

4. Albanese MA, Mejicano G, Mullan P, Kokotailo P, Gruppen L. Defining characteristics of educational competencies. Med Educ. 2008;42:248-55
5. Govaerts MJB. Educational competencies or education for professional competence? Med Educ. 2008;42:234-6.

6. Smith AF, Greaves JD. Beyond competence: defining and promoting excellence in anaesthesia. Anaesthesia. 2010;65:184-91.

7. Nocite JR. Recuperação pós-anestésica. Aspectos gerais. Rev Bras Anestesiol. 1987; 37(3):161-7.

8. Fernandes CR, Gomes JMA, Morais RP, Marinho DS, Holanda MA, Oliveira FRA. Avaliação sistemática do delirium e da dor em pacientes criticamente enfermos. Revista Dor. 2009;10:158-68.

9. Haynes AB, Weiser TG, Berry WR, Lipsitz SR, Breizat Abel-Hadi S, Dellinger EP, et al. A Surgical Safety Checklist to Reduce Morbidity and Mortality in a Global Population. N Engl J Med. 2009;360(5):491-9.

10. Haller G, Stoelwinder J, Myles PS, McNeil J. Quality and safety indicators in anesthesia: a systematic review. Anesthesiology. 2009;110:1058-75.

11. Seetharaman H. Ethical issues in anesthesia: the need for a more practical and contextual approach in teaching. J Anesth. 2009;23:409-12.

12. Epstein RM. Assessment in medical education. N Engl J Med. 2007;356(4):387-96

13. Fluit CRMG, Bolhuis S, Grol R, Laan R, Wensing M. Assessing the Quality of Clinical Teachers. A Systematic Review of Content and Quality of Questionnaires for Assessing Clinical Teachers. J Gen Intern Med [periodico na internet]. 2010 [acesso em 19 ago. 2010].

14. Dijkstra J, Van der Vleuten CPM, Schuwirth LWT. A new framework for designing programmes of assessment. Adv in Health Sci Educ. 2009;14:238-43.

15. Burch V, Norman G, Schmidt H, Van der Vleuten CPM. Are specialist certification examinations a reliable measure of physician competence? Advances in Health Sciences Education. 2008;13(4):521-33.

16. Kogan JR, Holmboe ES, Hauer KE. Tools for Direct Observation and Assessment of Clinical Skills of Medical Trainees: A Systematic Review. JAMA. 2009;302(12):1316-26.

17. Nothnagle M, Reis S, Goldman R, Diemers A. Development of the GPSE: a tool to improve feedback on procedural skills in residency. Fam Med. 2010;42(7):507-13.

18. Qu B, Zhao YH, Sun BZ. Evaluation of residents' competency in professionalism and communication skills in northern China: a 360-degree assessment. Med Teach. 2010;32(8):701-2.

19. Berk RA. Using the 360 multisource feedback model to evaluate teaching and professionalism Med Teach 2009;31:1073-1080. 
20. Buckley J, ColemanJ et al. The educational effects of portfolios on undergraduate student learning: A Best Evidence Medical Education (BEME) systematic review. BEME Guide No. 11. Medical Teacher. 2009;31:340-55.

21. Mann K, Gordon J, MacLeod A. Reflection and reflective practice in health professions education: a systematic review. Adv in Health Sci Educ. 2009;14:595-621.

22. Botti SHO, Rego S. Preceptor, Supervisor, Tutor e Mentor: Quais são os seus Papéis? Rev Bras Educ Med. 2008;32(3):363-73.

23. Kuhn CM.The Innovative Anesthesiology Curriculum. A Challenge and Hope for the Future. Anesthesiology. 2010;112:267-8.

24. Kopp VJ, Shafer A. Anesthesiologists and perioperative communication. Anesthesiology. 2000;93:549-55.

25. Slullitel A. Gestão da qualidade em anestesiologia. Prática Hospitalar. 2008;58(7/8):93-6.

26. Diego LA. Anestesiologia e Qualidade no Cuidado. Anestesia em Revista. 2009;59:18-25.

27. Gawande A. The checklist manifesto: How to get things right. New York: Metropolitan Books; 2010.

28. Mann K, Gordon AML. Reflection and reflective practice in health professions education: a systematic review. Adv in Health Sci Educ. 2009:14:595-62.

29. Driessen E. Portfolio critics: Do they have a point? 2009;31:317-9
30. Whitcomb ME. More on Competency-Based Education. Acad Med. 2004;79(6):493-4.

31. Santos WS. Organização Curricular Baseada em Competência na Educação Médica. Rev Bras Educ Med. 2011;35:86-92

\section{CONTRIBUIÇÃO DOS AUTORES}

Alberto Farias Filho coordenou o grupo de trabalho para elaboração do curriculo; Josenilia Maria Alves Gomes, participou do grupo de trabalho para elaboração do curriculo, colaborou com a elaboração do texto, análise crítica e revisão final; Washington Auspilicueta Pinto Filho, Glauco Kleming Florêncio da Cunha, Flavio Lobo Maia participaram do grupo de trabalho para elaboração do currículo

\section{CONFLITO DE INTERESSES}

Declarou não haver.

\section{ENDEREÇO PARA CORRESPONDÊNCIA}

Cláudia Regina Fernandes

Rua Marcelino Lopes, 4520, casa 9

Sapiranga - Fortaleza

CEP. 60833-075 CE

E-mail: crf@fortalnet.com.br 